\title{
Re-Thinking Diagnostic Classification of the Dysarthrias: A Developmental Perspective
}

\author{
A.T. Morgan $^{\mathrm{a}-\mathrm{c}}$ F. Liégeois ${ }^{\mathrm{d}}$

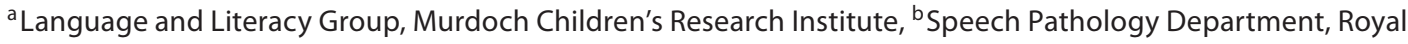 \\ Children's Hospital, and 'Department of Paediatrics, University of Melbourne, Melbourne, Vic., Australia; \\ dDevelopmental Cognitive Neuroscience Unit, University College London Institute of Child Health, London, UK
}

\section{Key Words}

Childhood dysarthria $\cdot$ Classification system .

Developmental considerations

\begin{abstract}
Acquired childhood dysarthria (ACD) receives little attention in the research literature in contrast with the adult correlate of the disorder. Speech language pathologists working in this field find diagnosis and management challenging, arguably because there is no child-based dysarthria diagnostic classification. Clinicians are either dependent upon developmental speech models that are not specific to dysarthria and that ignore the neural basis of the disorder, or on adultbased neurobehavioural classification systems. Here we consider the necessary elements for developing a clinically useful and empirically driven diagnostic classification system for ACD. The paper is divided into 2 parts. First, we question whether an adult diagnostic model can be validly applied to children. Second, we propose a methodological approach to develop a classification system for ACD. Specifically, we propose that advancing knowledge in neurobehavioural correlations of ACD is contingent upon large-scale studies, likely requiring international collaboration, which pool brain and speech outcome data. Ideally, researchers across centres would apply standard protocols to: (1) charac-
\end{abstract}

terize speech behaviour, and (2) brain structure, function and connectivity. When enough data is available to achieve statistical power, analysis could determine subgroups of dysarthria defined by speech behaviour. The commonalities of neural profiles of subgroups could then be examined to create an empirically driven theory of brain-behaviour relationships in ACD to underpin the classification system. Clinical diagnosis for children with ACD will remain limited until such data become available.

Copyright $\odot 2010$ S. Karger AG, Basel

\section{Introduction}

Attempts at classifying adult dysarthria have been made for over 65 years, arguably since the first attempt by Froeschels in 1943 [for review of the evolution of adult dysarthria classification systems, see 1]. There is consensus, however, that the most seminal contribution in this area has been the Mayo Clinic classification system (herein referred to as the Mayo system) provided by Darley, Aronson and Brown (DAB) $[2,3]$. To this day, their classification system linking brain pathology and speech behaviour remains the most commonly utilized for adultbased clinicians and researchers alike $[1,4-6]$. The longevity and popularity of the Mayo system is likely due in

A.T. Morgan

Language and Literacy Group

Murdoch Children's Research Institute

Melbourne, Vic. (Australia)

Tel. +61 39345 4215, Fax +61 39345 7041, E-Mail angela.morgan@mcri.edu 
part to its continual evolution. A multitude of adult-based studies have extended and refined the speech features originally reported by DAB. With advances in neuroimaging data acquisition and analysis techniques over the last decade, studies have also improved our understanding of the neural basis of adult acquired speech disorders [e.g. 7, 8].

In contrast, no such neurobehavioural classification system exists in acquired childhood dysarthria (ACD), despite the fact that dysarthria is a neurologically based disorder. This may be partly due in part to the limited research in the field of ACD relative to the adult literature. Further, existing paediatric-based studies have focused either on characterizing the speech behaviour, with a noticeable lack of attention given to the precise neural basis of the dysarthria, or on the neural basis, with little description of the speech characteristics of the disorder (e.g. using gross terminology such as 'dysarthria' $[9,10]$ or 'slurring of speech' [11]). Thus, in the absence of a paediatric-based system, clinicians are reliant on adult-based approaches, such as the Frenchay Dysarthria Assessment [12] or Mayo system (DAB), or on child-based models that do not consider neuropathology and are not specific to dysarthria [e.g. 13]. Recent data from 51 paediatric rehabilitation clinicians across Australia, New Zealand, the UK and southern Ireland confirmed that $67 \%$ of this group do indeed rely on the adult-based Mayo system for diagnosis of dysarthria [14]. Interestingly, this figure is comparable to the reported level of use of the Mayo system for dysarthria diagnosis (60\%) by 100 US-based adult clinicians and academics [6].

It is possible of course that the adult-based Mayo system may be appropriately applied to children. Some have suggested it is appropriate for use with children until evidence suggests otherwise [15]. However, enough preliminary data exists for us to now question whether childand adult-based features of the dysarthrias are comparable. Van Mourik et al. [16] were the first to challenge whether an adult classification system could be validly applied to children (see 'Part 1' for further detail).

Ten years on from the views expressed by van Mourik et al. [16], we call for further research exploring the validity of application of the adult model to children and question whether a child-based system is required [17, 18]. This paper therefore first considers whether an adult classification system can be validly applied to children. Second, we consider the necessary elements for developing a clinically useful and psychometrically robust diagnostic classification system for ACD that is underpinned by empirical data on brain and behaviour relationships.

Re-Thinking Diagnostic Classification of the Dysarthrias

\section{Part 1: Can an Adult-Based Dysarthria Classification System Be Applied to Children?}

First, let us examine the possible reasons why clinicians resort to using an adult classification system of dysarthria. Existing models of motor speech disorder in children provide a useful method for general categorization between disorders of planning/programming (i.e. childhood apraxia of speech) versus those of execution (i.e. developmental dysarthria) [e.g. 13]. However to our knowledge, no child-based system provides a subclassification of the dysarthrias beyond the level of 'developmental dysarthria'. Further, no models focus on acquired brain injury, and as such they include nil or minimal reference to the neural basis of the disorder.

In short, existing developmental models are not specific to dysarthria, and nor do they purport to be diagnostic classification systems. The absence of a neurobehaviourally based system is an obvious factor driving paediatric clinician's to utilize the adult-based DAB approach [14].

Next, we consider the application of the Mayo system to ACD. The authors concur with others who have written on the seminal contribution of the DAB model $[1,4$, 19]. It is important to mention that it was not $D A B$, but rather paediatric clinicians and researchers over time who have applied the Mayo system to children. Hence, this section does not aim to criticize the DAB model, but with a view to improving clinical practice in paediatrics, we critically question whether the application of this model to children is valid and empirically justified. Further, it is beyond the scope of this paper to discuss broader issues of the Mayo system that have been considered in the adult literature, including clinical utility and validity $[1,4-6,19]$, stimuli or task selection [20], intra- and inter-rater reliability [5], and effects of listener background and experience on rating [5]. Such issues would pose equal challenges in the paediatric field, but they have not been systematically examined in relation to children, and hence will not be further expanded upon here.

Two central themes will be addressed. Firstly, we consider from a theoretical and empirical basis whether it is appropriate to apply this adult model to children. Secondly, we discuss the deficiency in neural data accompanying motor speech profiles in children.

\section{Relevance of the Application of an Adult Model to Children}

To deduce the validity of applying the adult model to children, van Mourik et al. [16] reviewed the literature to 
determine whether distinct dysarthria types existed in children similar to those in adults. They examined Medline databases from 1980 to 1996 for publications in English using the search terms 'dysarthria' or 'speech disorders'. The authors then selected, reviewed and analysed studies of acquired dysarthria for children aged 16 years or less, and included studies mentioning perceptual judgement of speech features (according to the Mayo system) soon after the onset of the speech disorder. The review documented features of ACD associated with cerebellar lesions, brainstem lesions, basal ganglia lesions, cerebral cortical lesions: anterior opercular syndrome, and 'other' cerebral cortical and internal capsule lesions. It was reported that whilst basal ganglia lesions were rarely documented in children, they were the only group with highly similar speech features to adults. Children with cerebellar lesions, for example, did not demonstrate the pattern of 'excess and equal stress' commonly associated with ataxic dysarthria in adults. The authors did acknowledge however that the pathology underlying adult (e.g. spinocerebellar ataxias for example) and paediatric versions (cerebellar tumours) of ataxia differed, possibly explaining the varying outcomes. Overall however, it was concluded that ACD required its own classification system that should be developed through the detailed analysis of speech features in dysarthric children. Kent [4] also reiterated that despite similarities in some speech behaviour, it is not yet clear whether developmental and adult forms of dysarthria reflect the 'same mechanisms of impairment' (p. 412), and concurred with van Mourik et al. [16] that it may be unwise to apply the adult taxonomy to children, particularly in light of the fact that disorders affecting speech motor learning in children may differ from those that alter established speech motor skills in adults [4].

As mentioned above, an important consideration that limits application of the Mayo system to ACD is different pathology or aetiologies between adult-onset and childhood-onset dysarthrias. Some conditions and syndromes reported to be associated with speech disorders are developmental by nature, and there is therefore no adult equivalent. To name only a few, cerebral palsy [21], neural migration disorders (e.g. polymicrogyria [22]), epilepsyrelated disorders (e.g. Worster-Drought syndrome [23]) and progressive diseases with a childhood onset (e.g. Rasmussen's encephalitis, Sturge-Weber syndrome) are generally diagnosed in childhood. Finally, degenerative conditions included in the Mayo system, such as Parkinson's and Huntington's diseases, are extremely rare in children [for reports on juvenile forms of those conditions associated with dysarthria, see 24,25$]$.
Another major issue to consider when studying ACD is time of insult/onset of pathology. Unlike in adulthood where the speech mechanisms are fully mature, and therefore their neural basis relatively stable, the same cannot be said in children. One crucial factor is the supposedly higher potential for functional reorganization in the child relative to the adult brain (as seen for language [e.g. 26, 27]). The potential for functional reorganization of speech functions in childhood remains largely unexplored, mainly due to the dearth of data on large cohorts of children with similar insults at different ages. It is now accepted that brain plasticity for cognitive and motor functions varies widely after childhood-onset injuries, and does not necessarily decrease linearly with age. For instance, a large study on long-term cognitive outcome after traumatic brain injury indicated that children with earlier onset do worse than their late-onset counterparts, consistent with an 'early vulnerability' view rather than an 'early plasticity' view [28]. In the case of focal brain injury, in contrast, functional plasticity for cognitive functions may follow a U-shape [29]. Although spontaneous recovery of speech functions after childhood-onset dysarthria may be observed in numerous instances within the clinical setting, to our knowledge no large-scale study has documented its time course and the factors that may influence this time course, such as age at onset and aetiology. Although the problem of evolution of symptoms with time may be similar to that encountered after adult-onset stroke for instance, age at injury is an additional factor to take into account when considering ACD. Indeed, whether synaptogenesis, neural migration, functional, or structural changes in connectivity explain the evolution of recovery has to our knowledge never been addressed.

Furthermore in relation to the consideration of neural pathology, the DAB model preceded the consideration of hemispheric lateralization of speech functions, i.e. whether dysarthria types may differ between left and right hemispheric lesions. In recent times, functional MRI (fMRI) studies involving overt speech have reported a predominantly left cerebral hemisphere network for the planning and execution of speech movements in healthy controls [e.g. 30]. In line with this observation, a recent report on 62 adult cases in the acute phase [7] indicated that dysarthria may be more severe and more frequent after left compared to right hemisphere cerebral ischemia in adults. To our knowledge, no such study has been carried out in childhood cerebral infarction. Given the potential for inter-hemispheric compensation for language functions by the right hemisphere (even after removal of 
a whole cerebral hemisphere or hemispherectomy [31, 32]), the question of the potential difference between dysarthria caused by left and right hemisphere cerebral or cerebellar lesions in childhood may be of importance.

A final restriction of the Mayo system, regarding its application to ACD, is that the categorization of neuropathology associated with dysarthria subgroups is relatively gross by contemporary standards. At the time of development of the Mayo system, there was no capacity to sensitively define neural pathology. Due to the increasing versatility of MRI in present times however, we have an abundance of data acquisition and analysis techniques for visualizing and quantifying lesion location, and for understanding the relationship between brain structure, function and connectivity. In regard to data acquisition, high-resolution/high-contrast structural MR images are well recognized to provide detailed information at a macroscopic structural level. However, methods now exist that provide images based on brain function (e.g. fMRI) and on cellular processes occurring at a microscopic spatial scale (e.g. diffusion-weighted MRI). In addition, advances in statistically based image analysis tools (e.g. Statistical Parametric Mapping version 8, Wellcome Department of Neuroimaging, London, UK) enable abnormalities to be identified that are not detectable through visual inspection of images. These techniques are further outlined in 'Part 2' below.

\section{Part 2: Proposed Approach to Developing a Classification System for Children}

Synonyms for the term 'classify' include: categorize, catalogue, arrange, sort, or form a taxonomy. Each term varies subtly in its meaning, and the ways in which particular items can be classified are potentially interminable, regardless of the area of study. Hence, a successful classification system will be unambiguous regarding: the model or theory applied to classify the items, and the desired purpose of the classification (e.g. for clinical diagnosis, or for subgrouping individuals for enrolment in treatment studies).

Further, similar to a 'theory' in the empirical sense, a classification system should be falsifiable/testable, and therefore by definition the system will be in a constant state of development and revision as further empirical data becomes available. The system should also be the most parsimonious tool. Finally, the model should be 'rigorously tentative' and as close as possible to the current state of knowledge at any particular time. The inter- nationally applied Diagnostic and Statistical Manual of mental disorders (DSM-IV) is an example of a successful classification system. It meets the aforementioned criteria by being relatively simple, and it is updated as new research becomes available.

In light of these central tenets for classification systems, we have a working definition or explicit purpose for a diagnostic classification system for ACD: to provide patients with a behavioural speech diagnosis in conjunction with identifying behavioural symptoms that require treatment. This empirically driven classification system will be underpinned by a theory of brain-behaviour relationships. Given the little evidence of brain-behaviour data available in this field, we must develop the classification system and gather data on neural pathology in parallel. It is only when our evidence of the latter has reached a significant level that we can develop an empirically driven theory to underpin development of a neurobehavioural classification system. The necessary steps to achieving this aim are outlined in further detail below.

\section{Step 1: Develop a Paediatric Dysarthria Classification} System Based on Speech Features

A first step in developing a classification system is to collect perceptual, acoustic and physiological speech data [4] in large cohorts of patients with dysarthria associated with presumably similar aetiologies (syndromes, cerebral infract, traumatic brain injury). Statistical methods such as factor analyses (e.g. principal component, principal access factoring analyses, among others) enable one to summarize the structure of a set of variables or reduce a small amount of variables to a smaller set. This approach could be used to examine how deviant speech features 'cluster' together. That is, to determine which features are significantly associated together versus those features that are just 'noise' in particular groupings. This is critical as a major limitation in utility of the Mayo system is the lack of a clear distinction between features anticipated within versus across dysarthria subgroups [6]. Thus, the most difficult aspect of conducting differential diagnosis for dysarthria with the Mayo system is reportedly the degree of overlap in features across subgroups and/or the degree of variability within particular subgroups [6]. An almost insurmountable number of data points are required to enable robust dissociation between significantly associated features and noise. In this respect, although this would in principle be possible by aggregating data from numerous single-case studies, it is unlikely that different research groups would have used the same criteria to classify patients. Hence we argue that large-scale studies of 
ACD, likely requiring international collaborations, are required to achieve acceptable statistical power to achieve the above aim.

\section{Step 2: Examine the Neural Bases of Dysarthria}

\section{Subcategories with Neuroimaging}

Once dysarthria subcategories have been empirically defined based on speech behaviour, the next step is to examine commonalities in the neural correlates underlying these conditions. Numerous approaches can be used to identify commonalities in brain lesions within groups of patients within the same dysarthria subcategory, or to identify differences across subcategories of dysarthria. Only a few approaches will be discussed here. Using highresolution MRI scans, and even automated methods of lesion identification [33], frequency maps of lesion location ('lesion overlap' maps) can be obtained within a particular patient group sharing a common disorder. This approach has been successfully used to reveal brain lesions most likely to be associated with apraxia of speech in adults [34; for an alternative approach and limitations of lesion overlap studies, see 35]. Voxel-based methods, such as voxel-based morphometry, can identify morphological abnormalities in the grey and white matter that are not visible on visual examination. Again, group differences and commonalities can be revealed beyond obvious 'visible' lesion sites. Such an approach has for instance been used to identify subtle brain abnormalities associated with developmental disorders that have no obvious structural lesion correlates (e.g. childhood apraxia of speech [36]), as well as dysphagia in adults after stroke [37].

Perfusion imaging is also a promising tool for studying the neuroanatomical basis of ACD, especially in childhood stroke, where functional abnormalities can be detected remote from the original infarct (as in cortical regions after basal ganglia stroke [38]).

Since speech planning, programming and execution are subserved by networks of interconnected cortical and subcortical regions, examining the integrity of white matter tracts will also be of critical relevance to the study of the neural basis of subgroups of ACD. Diffusionweighted imaging (DWI) [for a review of the techniques, see 39] can be applied to track specific white matter pathways ('fibre tractography') or to derive local measures of white matter integrity (such as fractional anisotropy or mean diffusivity). These diffusion-based techniques have been successfully used in other neurological conditions. For instance, a study of 101 dysarthric adults after stroke [40] revealed that the most common site of abnormality,

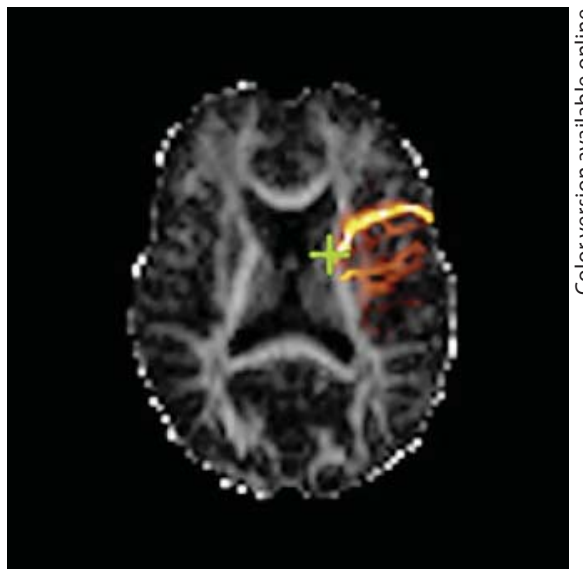

Fig. 1. Example of tractography of the left corticobulbar tract in a child with dysarthria following traumatic brain injury.

as revealed by DWI, was the corona radiata and the middle cerebral artery territory. In the motor domain, asymmetry of the pyramidal tract (measured with fractional anisotropy and mean diffusivity from DWI) has been found to correlate with the degree of motor impairment in patients with congenital hemiparesis [41]. Similar approaches could be used for the study of ACD, examining for instance the integrity of the corticobulbar tract (fig. 1), cerebellar, and basal ganglia control loops.

Although structural abnormalities of the brain can be the initial cause of ACD, there are cases where the speech disorder is a manifestation of a functional abnormality. Such functional abnormalities can be detected using functional MRI speech tasks. This is still an under-developed field in the study of childhood dysarthria, but has for instance been applied in the study of childhood apraxia of speech caused by a mutation of the FOXP2 gene in a British family [42]. Again, in future studies, fMRI may reveal regions that are functionally abnormal during dysarthric speech, remote from obvious lesion sites, and therefore help understand why seemingly distinct patient groups may fall within the same dysarthria subcategory.

Taken together, these MRI-based techniques examining brain structure, function and connectivity could be applied to the study of brain-behaviour relationships in ACD. The results could then be fed back to the behavioural classification system for the development of a neuroanatomical classification system of ACD (fig. 2). 
Fig. 2. Suggested approach to the development of a neurobehavioural classification system for ACD. Step 1 involves analysis of speech features for developing a speechbased diagnostic classification. In parallel, step 2 includes identifying neural correlates of dysarthria. Step 3 (white arrows) involves examining brain-behavioural correlations for the development of a neurobehavioural classification system.

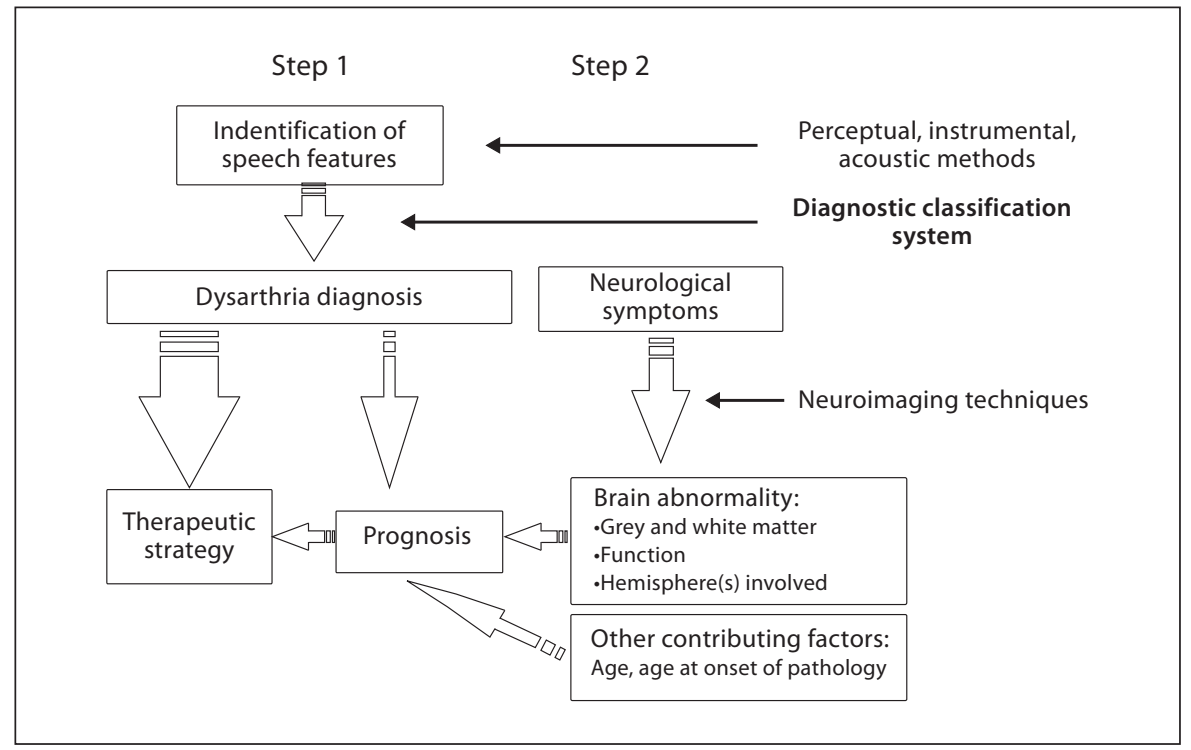

\section{Conclusion}

Clinicians currently working with ACD are reliant upon developmental motor speech classification models that are non-specific to acquired brain injury, or upon adult-based neurobehavioural models. Here we re-examined the appropriateness of applying an adult-based model to children, as raised previously by other researchers in this field. We conclude that further research is warranted to empirically determine child-based brain-behaviour profiles of ACD. Specifically, we suggest that advanced knowledge of the neurobehavioural correlations of ACD is contingent upon pooling brain and speech outcome data across large international collaborations. Ideally, researchers would apply: (1) standard detailed protocols to characterize speech outcomes, and (2) sophisticated neuroimaging data acquisition and analysis techniques that consider brain structure, function and connectivity to groups of children with similar aetiologies. Subgroups of dysarthria could then be determined, as defined by sharing common clusters of speech behaviour. The neural correlates for the dysarthria subgroups can then be examined to develop a brain-behaviour model for ACD that underpins the neurobehavioural diagnostic classification system. We suggest that a child-specific diagnostic model would yield more sensitive diagnosis and management, with a view to enhancing speech outcomes for these children.

\section{Acknowledgements}

Thank you to collaborators Dr. C. Clark and Dr. J. Clayden, UCL Institute of Child Health, UK, for tractography analysis and generation of figure 1. Dr. Morgan is funded by the National Health and Medical Research Council (\#607315) and the Victorian Neurotrauma Initiative (\#D131).

\section{References}

1 Duffy JR, Kent RD: Darley's contributions to the understanding, differential diagnosis, and scientific study of the dysarthrias. Aphasiology 2001;15:275-289.

-2 Darley FL, Aronson AE, Brown JR: Clusters of deviant speech dimensions in the dysarthrias. J Speech Hear Res 1969;12:462-496.

-3 Darley FL, Aronson AE, Brown JR: Differential diagnostic patterns of dysarthria. J Speech Lang Hear Res 1969;12:246-269.
4 Kent RD: Research on speech motor control and its disorders: a review and prospective. J Commun Disord 2000;33:391-428.

5 Bunton K, Kent RD, Duffy JR, Rosenbek JC, Kent JF: Listener agreement for auditory perceptual ratings of dysarthria. J Speech Lang Hear Res 2007;50:1481-1495.
6 Simmons KC, Mayo R: The use of the Mayo Clinic system for differential diagnosis of dysarthria. J Commun Disord 1997;30:117132.

7 Urban PP, Rolke R, Wicht S, Keilmann A, Stoeter P, Hopf HC, Dieterich M: Left-hemispheric dominance for articulation: a prospective study on acute ischaemic dysarthria at different localizations. Brain 2006;129: 767-777. 
-8 Duffy JR, Peach RK, Strand EA: Progressive apraxia of speech as a sign of motor neuron disease. Am J Speech Lang Pathol 2007;16: 198-208.

9 Chen Z, Zhu G, Lin J, Wu N, Feng H: Acute cerebral paragonimiasis presenting as hemorrhagic stroke in a child. Pediatr Neurol 2008;39:133-136.

10 Golomb MR, Weiss SK, Ibrahim SH, deVeber GA: A 15-year-old boy with central nervous system vasculopathy presenting with dysarthria-clumsy hand syndrome. J Child Neurol 2002;17:241-243.

-11 Agrawal A, Joharapurkar SR, Gharde P: Ischemic stroke in a child mistaken as functional disorder. Clin Neurol Neurosurg 2007; 109:876-879.

12 Enderby P: The Frenchay Dysarthria Assessment. San Diego, College Hill, 1983.

13 Hayden DA: Differential diagnosis of motor speech dysfunction in children. Clin Commun Disord 1994;4:118-147.

14 Morgan A, Skeat J: Evaluating service delivery for speech and swallowing problems following paedriatic brain injury: an international survey. J Eval Clin Pract, in press (accepted 4th December 2009).

15 Murdoch BE, Ozanne AE, Cross JA: Acquired childhood speech disorders: dysarthria and dyspraxia; in Murdoch BE (ed): Acquired Neurological Speech Language Disorder in Childhood. London, Taylor and Francis, 1990, p 309.

16 van Mourik M, Catsman-Berrevoets $\mathrm{C}, \mathrm{Pa}$ quier PF, Yousef-Bak E, van Dongen HR: Acquired childhood dysarthria: review of its clinical presentation. Pediatr Neurol 2007;4: 299-207.

17 Morgan A, Liégeois F, Vargha-Khadem F: Motor speech profile in relation to site of brain pathology: a developmental perspective; in Maassen B, van Lieshout P (eds): Speech Motor Control: New Developments in Basic and Applied Research. Oxford, Oxford University Press, 2010.

18 Morgan AT, Vogel AP: Intervention for childhood apraxia of speech. Cochrane Database Syst Rev 2008;3:CD006278.

-19 Kent RD, Duffy JR, Slama A, Kent JF, Clift A: Clinicoanatomic studies in dysarthria: review, critique, and directions for research. J Speech Lang Hear Res 2001;44:535-551.

20 Kent RD, Kent JF: Task-based profiles of the dysarthrias. Folia Phoniatr Logop 2001;52: 48-53.
21 Pennington L: Cerebral palsy and communication. Paediatr Child Health 2008;18:405409.

-22 Kuzniecky R, Andermann F, Guerrini R: Congenital bilateral perisylvian syndrome: study of 31 patients. The CBPS Multicenter Collaborative Study. Lancet 1993;341:608612.

23 Clark M, Carr L, Reilly S, Neville BG: Worster-Drought syndrome, a mild tetraplegic perisylvian cerebral palsy: review of 47 cases Brain 2000;123:2160-2170.

24 Anselm IA, Sweadner KJ, Gollamudi S, Ozelius LJ, Darras BT: Rapid-onset dystoniaparkinsonism in a child with a novel atpla3 gene mutation. Neurology 2009;73:400-401.

25 Waugh JL, Miller VS, Chudnow RS, Dowling MM: Juvenile Huntington disease exacerbated by methylphenidate: case report. J Child Neurol 2008;23:807-809.

26 Mosch SC, Max JE, Tranel D: A matched lesion analysis of childhood versus adult-onset brain injury due to unilateral stroke: another perspective on neural plasticity and recovery of social functioning. Cogn Behav Neurol 2005;18:5-17.

27 Liegeois F, Connelly A, Cross JH, Boyd SG, Gadian DG, Vargha-Khadem F, Baldeweg T: Language reorganization in children with early-onset lesions of the left hemisphere: an fMRI study. Brain 2004;127:1229-1236.

-28 Anderson V, Catroppa C, Morse S, Haritou F, Rosenfeld J: Functional plasticity or vulnerability after early brain injury? Pediatrics 2005;116:1374-1382.

29 Anderson V, Spencer-Smith M, Leventer R, Coleman L, Anderson P, Williams J, Greenham M, Jacobs R: Childhood brain insult: can age at insult help us predict outcome? Brain 2009;132:45-56.

-30 Riecker A, Mathiak K, Wildgruber D, Erb M, Hertrich I, Grodd W, Ackermann H: fMRI reveals two distinct cerebral networks subserving speech motor control. Neurology 2005;64:700-706.

31 Liegeois F, Connelly A, Baldeweg T, VarghaKhadem F: Speaking with a single cerebral hemisphere: fMRI language organization after hemispherectomy in childhood. Brain Lang 2008;106:195-203.
32 Liegeois F, Cross JH, Polkey C, Harkness W, Vargha-Khadem F: Language after hemispherectomy in childhood: contributions from memory and intelligence. Neuropsychologia 2008;46:3101-3107.

33 Seghier ML, Ramlackhansingh A, Crinion J, Leff AP, Price CJ: Lesion identification using unified segmentation-normalisation models and fuzzy clustering. Neuroimage 2008;41: 1253-1266.

-34 Dronkers NF: A new brain region for coordinating speech articulation. Nature 1996;384: 159-161.

>35 Hillis AE, Work M, Barker PB, Jacobs MA, Breese EL, Maurer K: Re-examining the brain regions crucial for orchestrating speech articulation. Brain 2004;127:14791487.

36 Belton E, Salmond CH, Watkins KE, VarghaKhadem F, Gadian DG: Bilateral brain abnormalities associated with dominantly inherited verbal and orofacial dyspraxia. Hum Brain Mapp 2003;18:194-200.

-37 Gonzalez-Fernandez M, Kleinman JT, Ky PKS, Palmer JB, Hillis AE: Supratentorial regions of acute ischemia associated with clinically important swallowing disorders: a pilot study. Stroke 2008;39:3022-2028.

38 Rowan A, Vargha-Khadem F, Calamante F, Tournier JD, Kirkham FJ, Chong WK, et al: Cortical abnormalities and language function in young patients with basal ganglia stroke. Neuroimage 2007;36:431-444.

39 Schaefer PW, Ellen Grant P, Gilberto Gonzalez R: Diffusion-weighted MR imaging of the Brain. Radiology 2000;217:331-345.

40 Kumral E, Celebisoy M, Celebisoy N, Canbaz DH, Calli C: Dysarthria due to supratentorial and infratentorial ischemic stroke: a diffusion-weighted imaging study. Cerebrovasc Dis 2007;23:331-338.

41 Glenn OA, Ludeman NA, Berman JI, Wu YW, Lu Y, Bartha AI, Vigneron DB, Chung SW, Ferriero DM, Barkovich AJ, Henry RJ: Diffusion tensor MR imaging tractography of the pyramidal tracts correlates with clinical motor function in children with congenital hemiparesis. Am J Neuroradiol 2007;28: 1796-1802

42 Liegeois F, Baldeweg, T, Connelly A, Gadian DG, Mishkin M, Vargha-Khadem F: Language fMRI abnormalities associated with FOXP2 gene mutation: Nat Neurosci 2003;6: 1230-1237. 